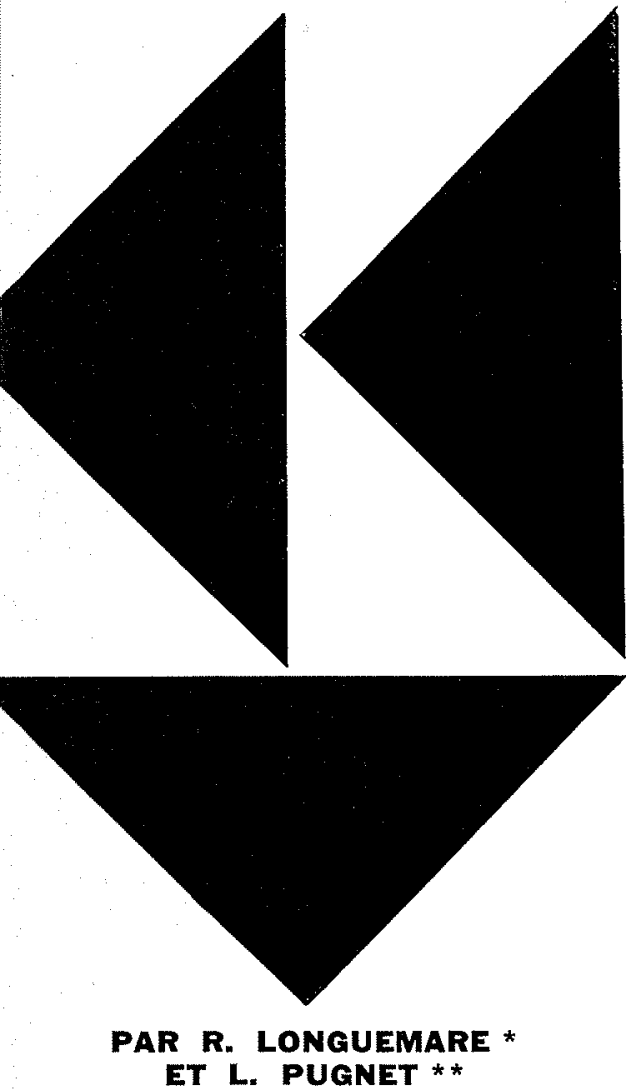

ET L. PUGNET * *

\section{CONSIDERATIONS SUR LES DECHARGEURS EN EXPLOITATION ET EN PROJET DES USINES DE LA BASSE-DURANCE}

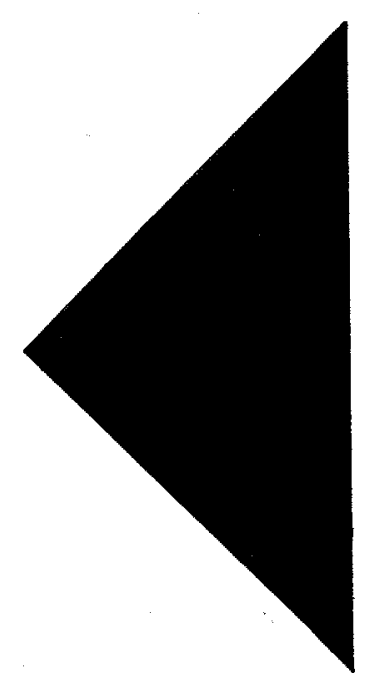

\section{Introduction}

Le bassin de la Durance constitue, avec ceux du Rhône et du Rhin, l'un des meilleurs gisements énergétiques français, puisque la puissance totale installée sera, à terme, voisine de 1 million et demi de $\mathrm{kW}$ pour une production globale annuelle moyenne de 6 milliards de $\mathrm{kWh}$, soit environ le $1 / 7^{\circ}$ de la production hydraulique actuelle (fig. 1). On distingue d'une part, la Basse-Durance et SerrePonçon, liés par le législateur en 1955 du fait de leur vocation agro-industrielle, et d'autre part la moyenne Durance en cours d'équipement avec un projet de 7 chutes. A l'aval de la cluse de Mirabeau, 85000 ha de riches terres sont irriguées et les apports de la Durance atteignent à cet endroit 6 milliards de $\mathrm{m}^{3}$ d'eau par an en moyenne, dont 5 sont utilisables (fig. 2). La mer n'est plus qu'à $45 \mathrm{~km}$ à vol d'oiseau et cette chute de $256 \mathrm{~m}$ sera exploitée par 5 usines échelonnées le long d'un canal de dérivation de $85 \mathrm{~km}$ de longueur, capable de transiter $250 \mathrm{~m}^{3} / \mathrm{s}$. Ce canal suit la R. G. de la Durance sur $50 \mathrm{~km}$, de Cadarache à Mallemort, en reliant les usines de Jouques, Saint-Estève-Janson, Mallemort: il s'en écarte ensuite pour rejoindre par le seuil de Lamanon, létang de Berre, en alimentant au passage les usines de Salon et de Saint-Chamas (fig. 3). Au total, la Basse-Durance apportera à elle seule une puissance installée de plus d'un demi-million de $\mathrm{kW}$ et une productibilité de 2,3 milliards de kWh. Les usines de Jouques et Saint-Estève ont été mises en service respectivement en 1959 et 1963. La mise en service de Salon et Saint-Chamas est pré-

* Ingénieur à E.D.F, R.E.H. ALPes-III.

* Ingénieur à E.D.F., C.R.E.C. vue pour le début de l'année 1966 . Celle de Mallemort devrait intervenir en 1971.

En première partie de cet exposé, nous rappellerons les caractéristiques des ouvrages en service (barrage et prise de Cadarache, chutes de Jouques et Saint-Estève) en indiquant les enseignements tirés de leur exploitation qui ont influencé les projets des chutes aval (barrage de Mallemort, chutes de Salon et Saint-Chamas).

La deuxième partie sera essentiellement consacrée aux études sur modèle des projets des déchargeurs des usines aval de Salon et Saint-Chamas, effectuées au Laboratoire de Chatou.

\section{Première partie}

\section{Barrage de Cadarache (fig. 4)}

Le barrage et la prise de Cadarache, point de départ du canal de la Basse-Durance, ont été présentés dans la communication de M. Fauconnier de juin 1957 sur «Le problème des affouillements à l'aval des barrages $\gg$. Situé près de la rive gauche, au confluent du Verdon avec la Durance, le barrage a 5 passes de $25 \mathrm{~m}$ de large, obturées par des vannes-segments de $6,50 \mathrm{~m}$ de hauteur; il comporte un dégraveur de fond de $5 \mathrm{~m}$ de largeur destiné à évacuer les matériaux de charriage devant les grilles de la prise. Le barrage est relié à la rive droite par une digue d'entonnement insubmersible. Il crée une retenue d'exhaussement à la cote 256 , permettant la dérivation d'un débit de $250 \mathrm{~m}^{3} / \mathrm{s}$ dans le canal d'amenée de l'usine de Jouques.

Les radiers ${ }^{\circ s} 1,4$ et 5 ont été réalisés en béton brut à l'oxydociment, le $n^{\circ} 2$ à l'aciérite, le $n^{\circ} 3$ à l'oxydociment, mais meulé. Malheureusement, les temps de fonctionnement des différentes vannes ont 

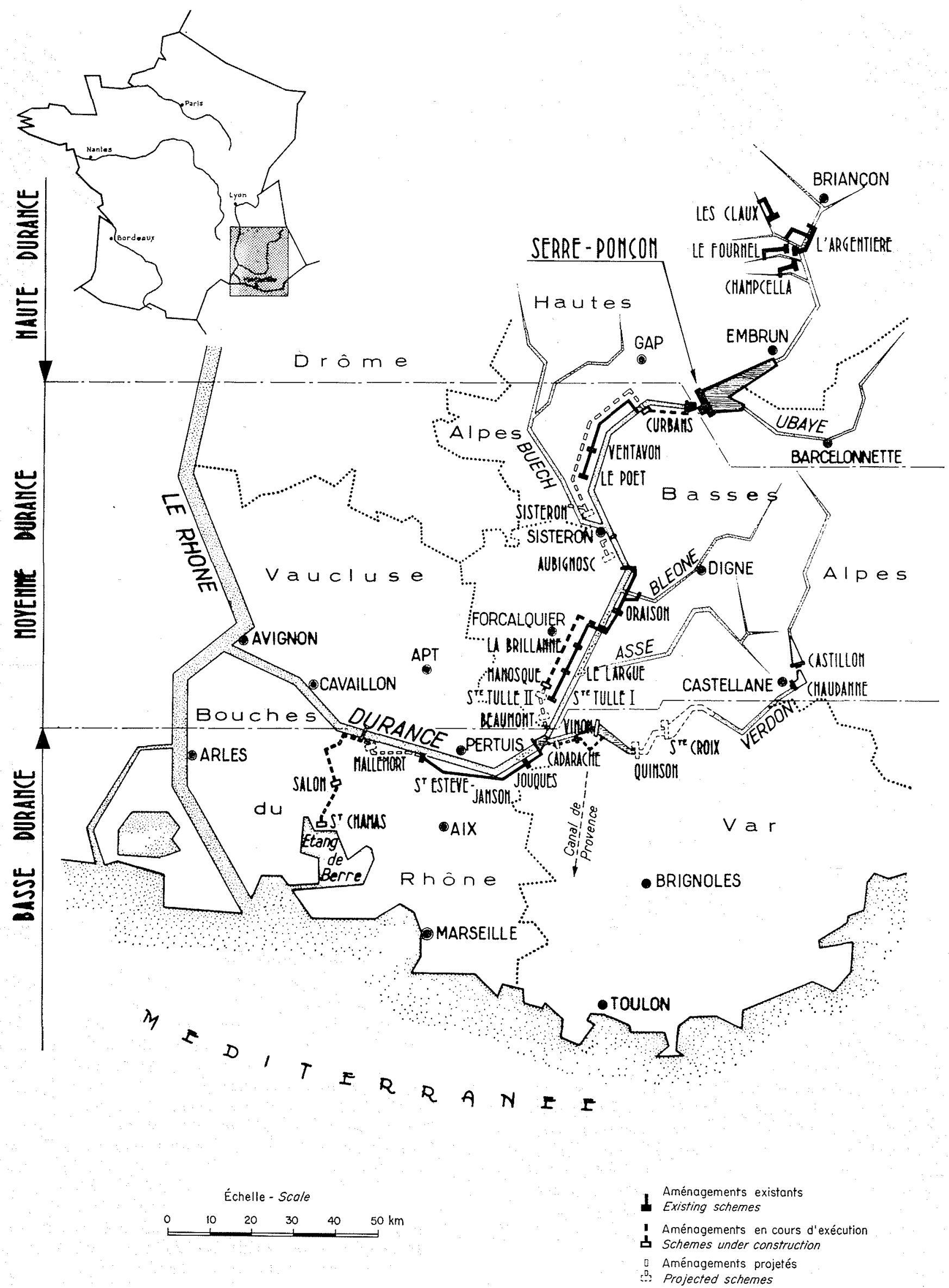

Bassin de la Durance. Plan général /1/ The Durance basin. General outline 
été notés seulement depuis l'an dernier, si bien qu'il est difficile de tirer des conclusions de la visite des passes du barrage en février 1964. D'une façon générale, les radiers ont bien résisté. Par contre, la rugosité artificielle de dissipation d'énergie constituée par des blocs en béton armé de $0,50 \times 0,50 \mathrm{~m}$ de section et de hauteur décroissante de l'amont vers l'aval, a subi des dégradations justifiant les craintes exprimées par M. l'Inspecteur général Aubert, lors de la discussion qui suivit la communication de M. Fauconnier. Cette rugosité comprend au fond de la cuvette de la passe, 4 rangées de blocs de $1 \mathrm{~m}$ suivies de 3 rangées de bloes de $0,50 \mathrm{~m}$ de hauteur sur la contre-pente aval du radier. Les petits blocs aval sont intacts; par contre, de nombreux blocs de $1 \mathrm{~m}$ ont été arrachés du radier ou cassés à la base ou à mi-hauteur. Dans la passe qui a le plus souffert, la $\mathrm{n}^{\circ} 2,36$ blocs sur 58 sont détruits, alors que la $n^{\circ} 5$ est intacte; au total, on a dénombré la destruction de 76 blocs sur 290. Les dégradations ont été causées par la cavitation, les obstacles étant insuffisamment noyés à certains régimes d'ouvertures des vannes et par les chocs des galets et des arbres que charrie la Durance en crue.

On peut se demander si la disparition d'une partie de la rugosité artificielle ne risquerait pas d'entraîner des dégâts à l'aval, du fait qu'une partie de l'énergie hydraulique n'est plus dissipée sur les radiers du barrage. A titre de contrôle, des profils en long du lit à l'aval du barrage ont été relevés en septembre 1959 après la mise en eau, en janvier 1961 et en mai 1964; les deux crues les plus importantes (pointe supérieure à $2000 \mathrm{~m}^{3} / \mathrm{s}$ ) ont eu lieu en octobre 1960 et en novembre 1963. Ces relevés permettent de ne constater aucun déchaussement a l'aval des radiers. Les points bas des affouillements se situent environ à $20 \mathrm{~m}$ à l'aval du barrage et atteignent au maximum : $4 \mathrm{~m}$ à l'aval de la passe $\mathrm{n}^{\circ} 1$ et $5 \mathrm{~m}$ à l'aval du dégraveur. A l'aval des autres passes, les fosses de 1 à $2 \mathrm{~m}$ de profondeur semblent avoir atteint leur profil d'équilibre.

Barrage de Mallemort (fig. 5)

Mis en service à la fin de 1964 , le barrage et la prise de Mallemort assureront la dérivation des eaux de la Durance vers les usines de Salon et Saint-Chamas dès le début de 1966 et jusqu’à la mise en service en 1971 de l'usine de Mallemort. A cette date, l'ouvrage servira à compenser les débits agricoles perdus entre Cadarache et Mallemort. En raison du caractère provisoire de cette phase d'exploitation, on a recherché, par de légers sacrifices sur la production d'énergie, des économies substantielles sur les ouvrages. Le barrage comprend 10 passes de $25 \mathrm{~m}$ de largeur obturées par des vannes-segments; 3 , dites principales, dont le seuil est à la cote 113,25 , assurent le réglage de la retenue à la cote 118,50 ; les 7 autres, dites courantes, dont le seuil est à la cote 114,25, ne s'ouvrent que lorsque le débit de la Durance dépasse $500 \mathrm{~m}^{3} / \mathrm{s}$ afin de rétablir la ligne d'eau naturelle. Le charriage ne commençant qu'à partir de ce débit, il était inutile de prévoir un dégraveur. La dissipation d'énergie hydraulique n'a lieu qu'aux ouvertures partielles des vannes principales, par ressaut hydraulique dans les cuvettes suffisamment profondes des passes, sans l'aide de rugosité artificielle. Les études sur modèle à l'échelle du $1 / 50^{\mathrm{e}}$ ont été effectuées au Laboratoire des Milles, près d'Aix-en-Provence.

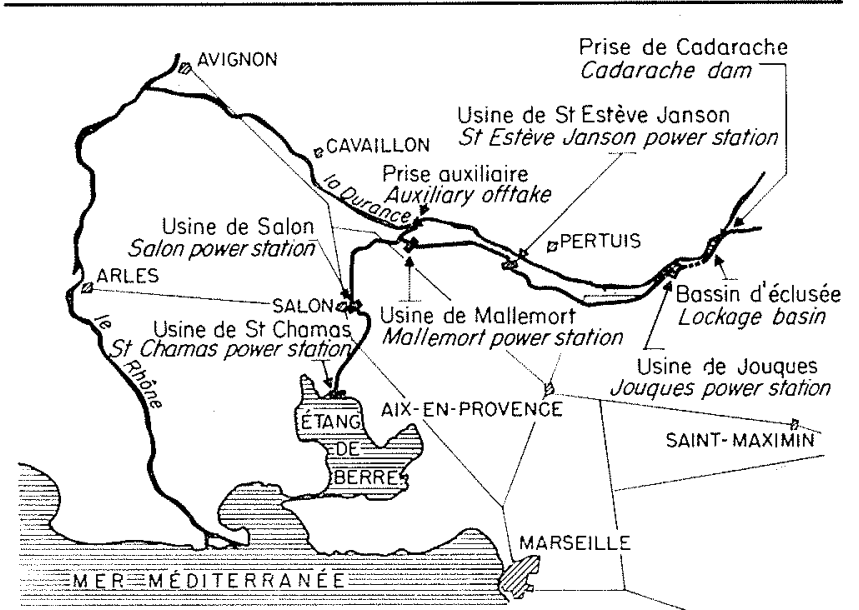

2/ Aménagement de la Basse Durance. Plan général. The Lower Durance hydro-electric scheme. General ontline.

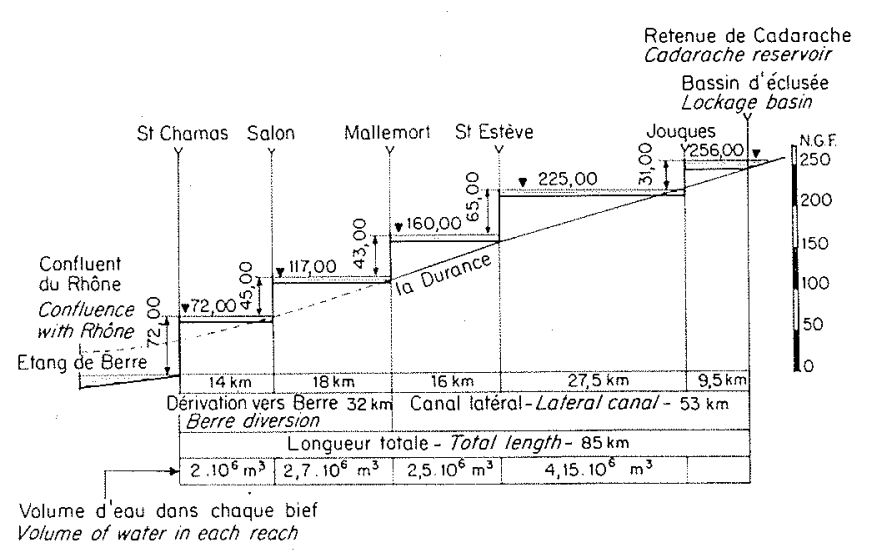

3/ Profil en long de la Basse Durance.

Lower Durance bed profile.

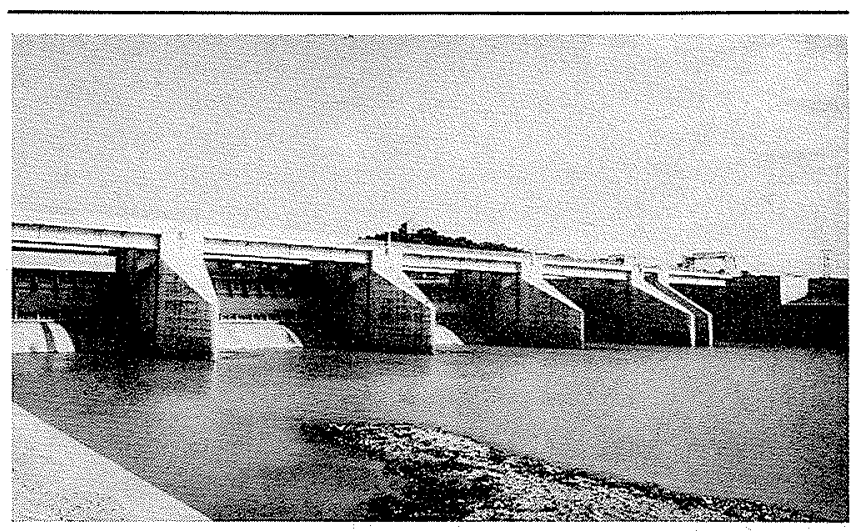

4) Barrage de Cadarache, vue de la R.D. aval. Cadarache dam seen from the right bank downstream.

5/ Barrage de Mallemort, vue de la R.D. amont. Mallemort dam seen from the right bank upstream.

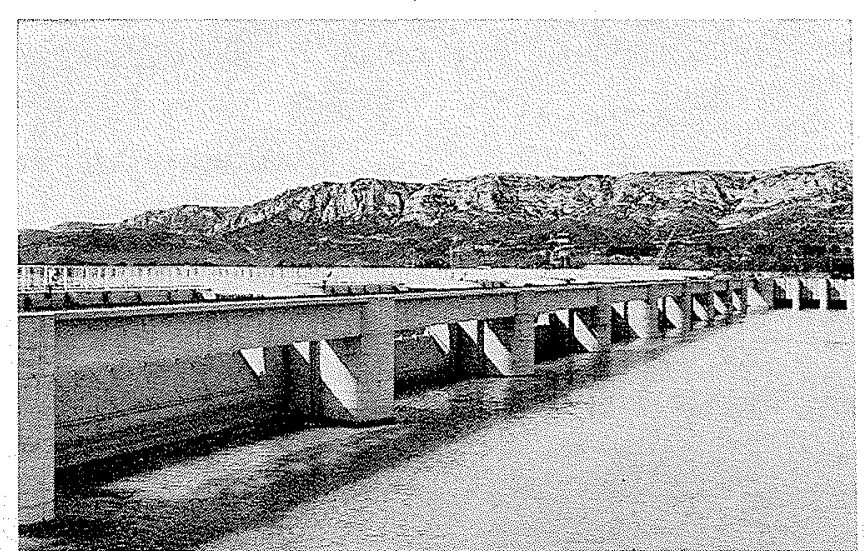




\section{Déchargeur de Jouques (fig. 6)}

Le déchargeur de Jouques est constitué par 3 pertuis en charge branchés sur la galerie d'amenée et obturés par 3 vannes-segment asservies aux groupes de l'usine (hauteur de chute équipée: $31 \mathrm{~m}$ ). Un coursier parabolique de $14 \mathrm{~m}$ de largeur guide les veines à grande vitesse sur une trentaine de mètres environ dans un bassin d'amortissement équipé d'une rugosité artificielle et d'un seuil déversant. La rugosité est constituée de 11 dents triangulaires de 2,20 $\mathrm{m}$ de hauteur et 1,20 m d'épaisseur suffisamment immergées, sous 9 mètres d'eau, pour éviter la cavitation. Une visite de l'ouvrage eut lien en septembre 1962, après un fonctionnement exceptionnellement important pour ce genre d'ouvrage (plus de 1000 heures), durant les 4 premières années, bien supérieur à son utilisation normale; aucune détérioration grave des dents n'a été décelée si ce n'est une légère usure des arêtes du béton et une certaine rugosité du radier présentant quelques trous peu profonds.

\section{Déchargeur de Saint-Estève (fig. 7)}

Le déchargeur de Saint-Estève est un ouvrage plus important de par la hauteur de chute équipée $(65 \mathrm{~m})$ et par la configuration topographique à l'emplacement de l'usine; il a plus de $300 \mathrm{~m}$ de long pour une largeur de $19 \mathrm{~m}$ et comporte, en plus des 3 pertuis de fond, un déversoir latéral de sécurité de $95 \mathrm{~m}$ de longueur. L'amortissement de l'énergie hydraulique et la stabilité des veines liquides sur le coursier posèrent des problèmes délicats qui furent résolus sur modèle (le lecteur se reportera à la communication de MM. Lamblé et Peutot, de mars 1958).

Rappelons que la stabilité de l'écoulement et la dissipation d'une grande partie de l'énergie sur le coursier du déchargeur furent obtenus à l'aide d'une rugosité artificielle constituée par des blocs de $0,60 \times 0,60 \mathrm{~m}$ de section sur $0,15 \mathrm{~m}$ de hauteur. Mais la réalisation de ces 2000 obstacles fut assez délicate sur le chantier malgré leur préfabrication. Compte tenu des fortes vitesses de l'écoulement, le béton des obstacles, du radier et des bajoyers fut protégé par 5 couches de peinture néoprène. Après un fonctionnement intense du déchargeur, durant la première année d'exploitation de l'usine, de nombreuses dégradations ont été constatées, notamment dans la partie amont du coursier dans les premières rangées de dents. Le néoprène et, par endroits, le béton ont été attaqués par cavitation et par érosion des graviers entraînés par l'écoulement. Pour ces raisons, le déchargeur de Salon ne comprendra que des rainures longitudinales coulées sur place dont le comportement en exploitation sera une expérience intéressante (fig. 8). Celui de SaintChamas ne comportera aucune rugosité; au contraire, on utilisera les fortes vitesses de l'écoulement pour déverser en saut de ski dans l'étang de Berre (fig. 9).

\section{Deuxième partie}

Les déchargeurs de Salon et Saint-Chamas ont été mis au point au Laboratoire National d'Hydraulique de Chatou, sur deux modèles à l'échelle du $1 / 30^{\circ}$. Le débit d'équipement des usines est de $250 \mathrm{~m}^{3} / \mathrm{s}$ et leurs hauteurs de chutes brutes $45 \mathrm{~m}$ et $72 \mathrm{~m}$ respectivement.
Les problèmes étudiés sur les modèles concernent les écoulements à grande vitesse dans les canaux à forte pente et la dissipation de l'énergie des chutes.

\section{Déchargeur de Salon}

Le déchargeur de Salon comporte (fig. 10):

- des organes d'alimentation, composés de trois pertuis de fond et d'un déversoir latéral de sécurité suivi d'un collecteur des eaux déversées;

- un canal de décharge;

- un bassin d'amortissement;

- des ouvrages de restitution au canal de fuite.

\section{LES ORGANES D'ALIMENTATION :}

Leur mise au point sur modèle n'a présenté aucune particularité et il suffit d'indiquer leurs caraetéristiques générales :

- les trois pertuis de fond sont équipés de vannessegment en charge, à manouvre rapide.

Chaque vamne est asservie à un groupe, afin d'assurer la continuité du débit dans les biefs amont et aval;

- le déversoir latéral de sécurité a une longueur de $105 \mathrm{~m}$ et évacue le débit de $250 \mathrm{~m}^{3} / \mathrm{s}$ sous la charge de $1,08 \mathrm{~m}$.

\section{LE CANAL DE DÉCHARGE :}

Long de $120 \mathrm{~m}$ et large de $19 \mathrm{~m}$, ce canal est alimenté dissymétriquement par les 3 pertuis de fond sur sa moitié rive gauche et par le déversoir latéral de sécurité sur sa moitié rive droite. Le projet initial comportait, au raccordement des pertuis de fond el du canal collecteur avec le canal de dé. charge, un changement de pente engendrant des perturbations dans l'alimentation du canal de décharge. Cette discontinuité a été supprimée en augmentant la pente de 12,9 à $14,4 \%$ de la partie amont du canal de décharge. Cette modification a permis de réduire le guideau de $10 \mathrm{~m}$ de longueur qui séparait initialement le canal collecteur et les pertuis de fond, à un arrière-bec profilé de $3,50 \mathrm{~m}$. Les arrière-bees des piles intermédiaires séparant les pertuis de fond, ont également été profilés (fig. 11).

Le coursier à l'aval de cette partie rectiligne présente un profil plongeant parabolique destiné à faciliter la pénétration de la veine d'eau dans le bassin d'amortissement.

Les modifications relatives aux ouvrages d'alimentation ne permettaient cependant pas d'obtenir un écoulement satisfaisant dans le canal de décharge. La mauvaise répartition des vitesses à la partie amont engendrant dans le canal des ondes stationnaires entraînant des débordements en différents points des bajoyers, dont la position variait avec les conditions d'alimentation (fig. 12).

La recherche de dispositifs de régularisation de l'écoulement et éventuellement de dissipation d'énergie a conduit à équiper successivement le coursier du canal de décharge par des dispositifs à dents (type Saint-Estève), à guideaux longitudinaux, à guideaux à chevrons (fig. 13).

Les dents du type Saint-Estève ont été disposées sur une longueur de $85 \mathrm{~m}$ de coursier; elles avaient en plan la forme d'un carré de $0,6 \mathrm{~m}$ de côté et une hauteur de $0,15 \mathrm{~m}$. Le dispositif à guideaux longitudinaux s'étendait sur. une longueur de coursier de $110 \mathrm{~m}$ et se composait de guideaux de $0,6 \mathrm{~m}$ de 

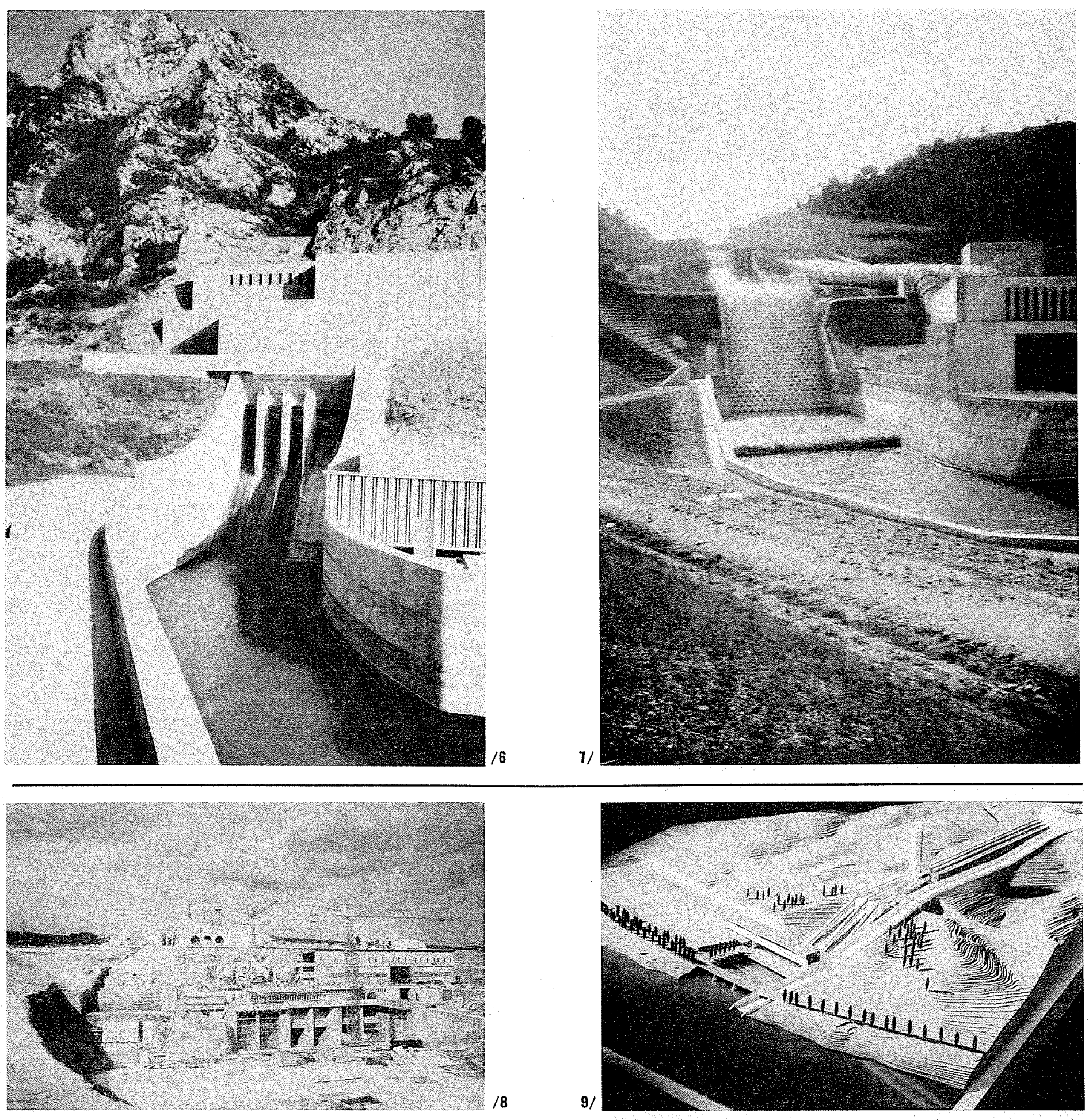

6/ Déchargeur de Jouques.

The Jouques spillway.

1/ Déchargeur de Saint-Estève. The Saint-Estive spillway.

8/ Usine et déchargeur de Salon, en construction. Salon power station and spillway during construction.

9/ Maquette de la chute de Saint-Chamas. Model of the Saint-Chamas scheme.

10/ Le déchargeur de Salon. General spillway arrangements at Salon.
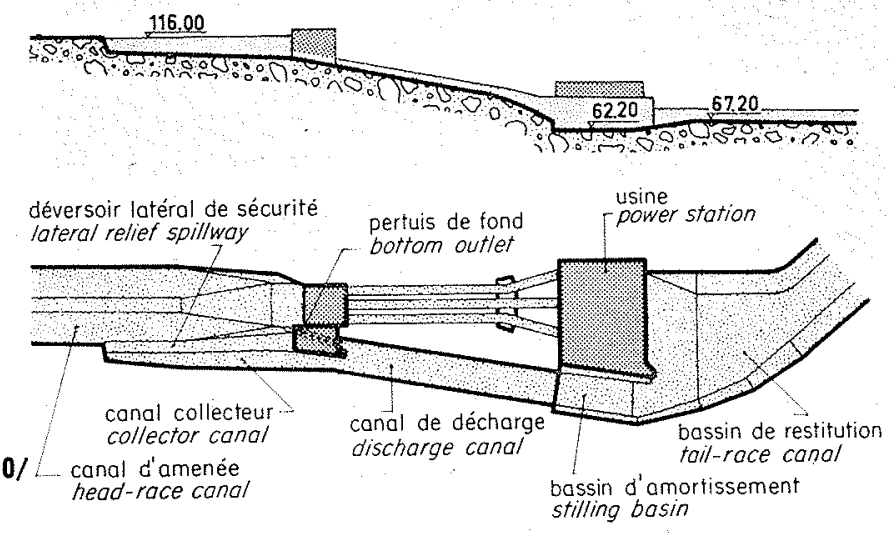


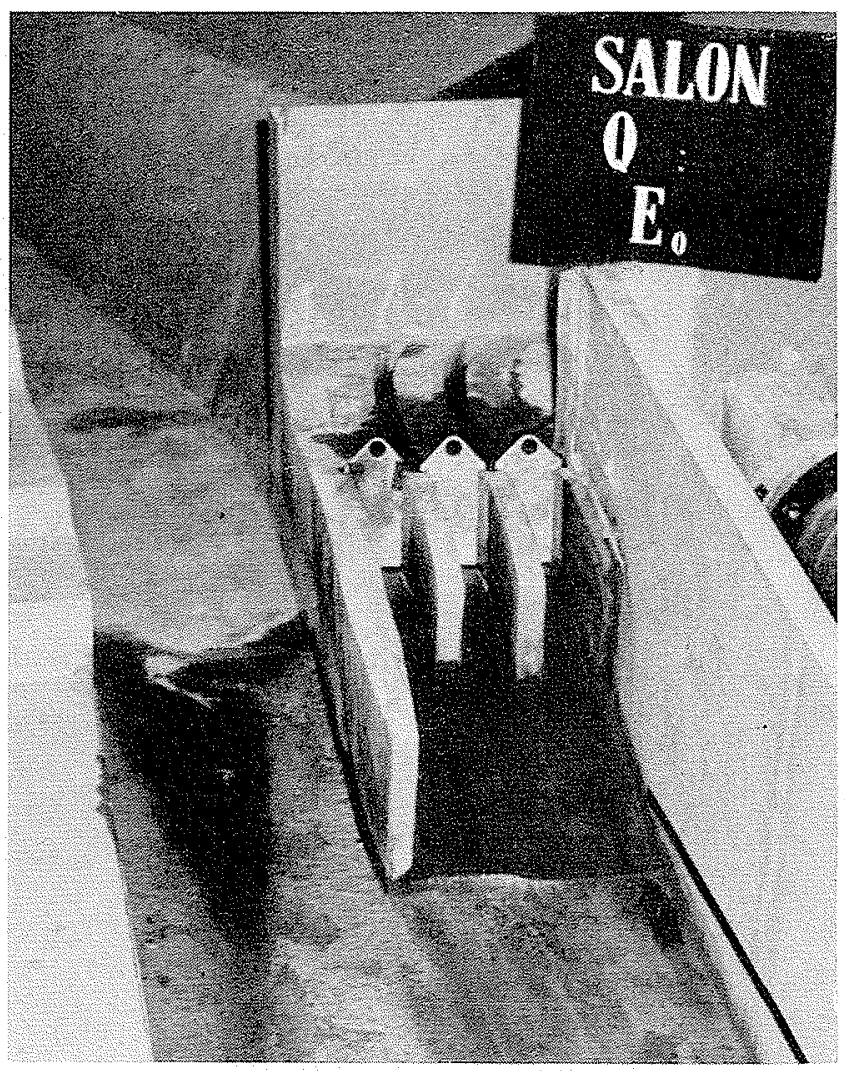

$111 /$

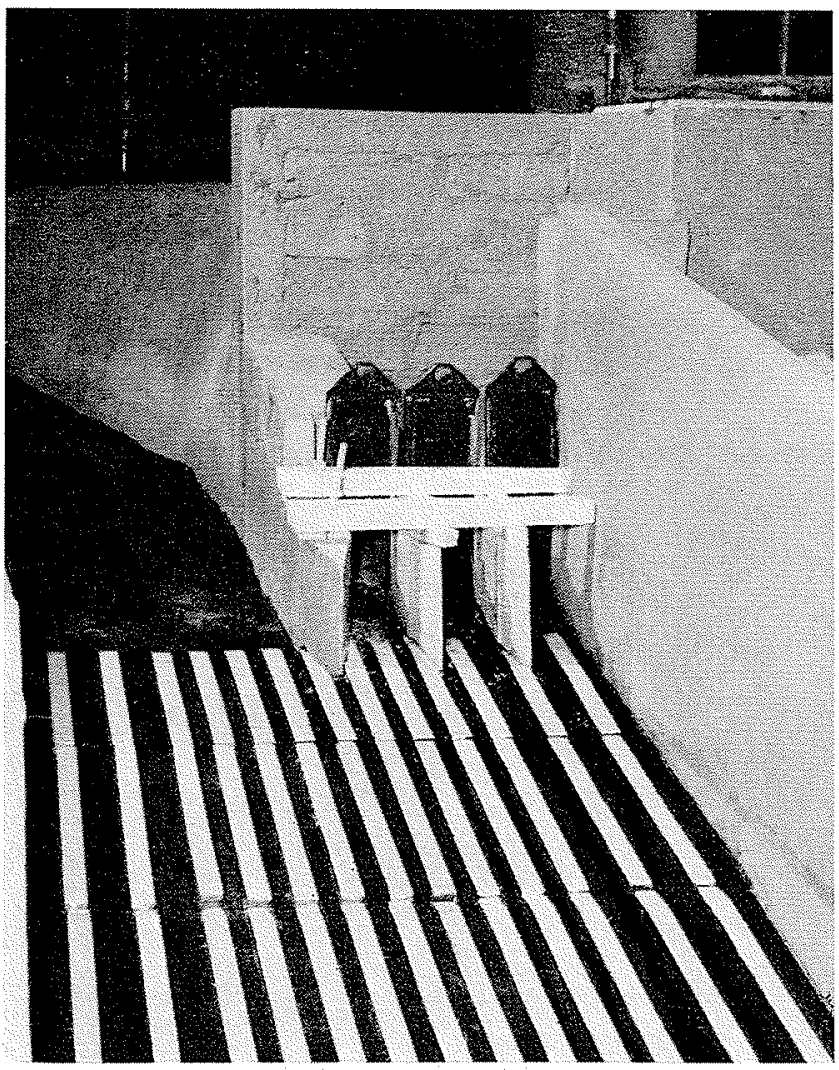

Projet initial

Initial design
Alimentation du canal de décharge de Salon. Discharge canal intakes arrangements at Salon.
Projet définitif Final design

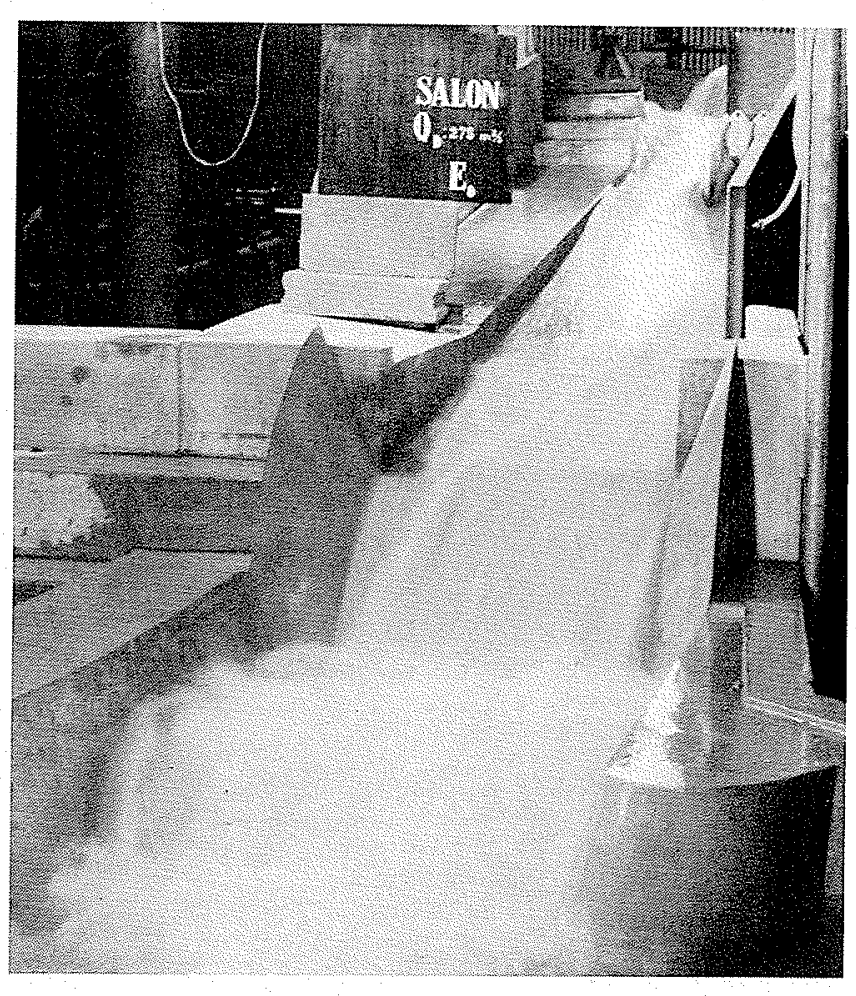

12) Ecoulement dans le canal de décharge de Salon. Projet initial.

Flow pattern in the Salon discharge canal. Initial design.

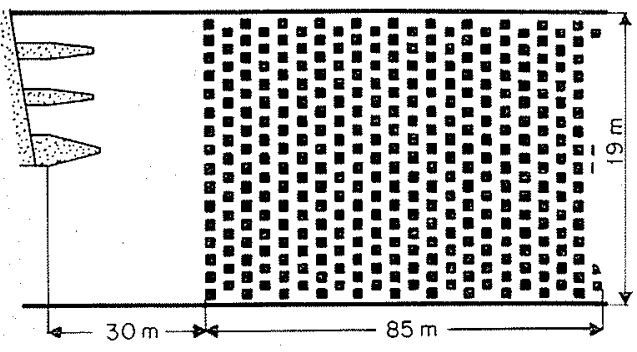

GUIDEAUX GROOVES

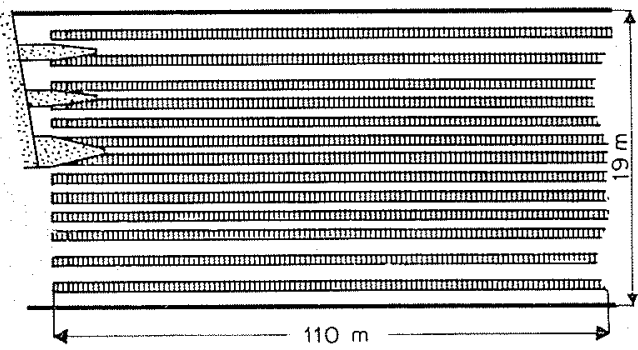

GUIDEAUX CHEVRONS

GROOVES $A N D$

CHEVRONS

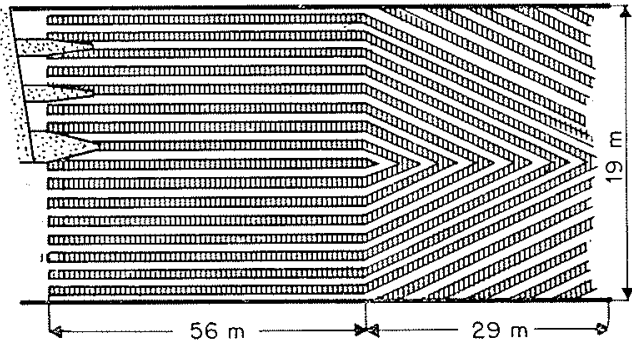

13/ Dispositif́s de régularisation de l'écoulement envisagés dans le canal de décharge de Salon.

Various tippes of flow control arrangements considered for Salon discharge canal. 
largeur et d'espacement: leur hauteur était de $0,24 \mathrm{~m}$. Le dispositif à guideaux et chevrons, d'une longueur totale de $85 \mathrm{~m}$ (dont $29 \mathrm{~m}$ pour les chevrons), comportait les mêmes guideaux que dans le dispositif précédenl; les chevrons se présentaient comme des guideaux inclinés à $45^{\circ}$ par rapport à la direction de l'écoulement. Les essais des trois dispositifs ont conduit à une régularisation acceptable de l'écoulement, sans toutefois, dans le cas des deux premiers, éliminer complèlement les ondes stationnaires.

Le relevé des vitesses longitudinales à la naissance de la partie plongeante du coursier a montré que la meilleure distribution était obtenue avec le dispositif à guideaux longitudinaux et que les vitesses les plus faibles, correspondant à la dissipation d'énergie la plus importante, étaient obtenues avec le dispositif à dents. Il reste en effet sous forme cinétique, dans la section de mesure :

- $45 \%$ de l'énergie de la chute dans le cas du coursier lisse;

- $25 \%$ dans le cas du dispositif à dents;

- $40 \%$ dans le cas du dispositif à guideaux et chevrons;

- $35 \%$ dans le cas du dispositif à guideaux longitudinaux.

La solution à guideaux longitudinaux a été retenue de préférence au dispositif à dents, plus efficace, mais de réalisation plus difficile. Une légère modification a toutefois été apportée par la Région d'Equipement à la disposition des guideaux afin de faciliter leur réalisation sur te chantier (fig. 14). La figure 15 montre qu'il subsiste encore des ondes stationnaires dans le canal, mais d'amplitude acceptable.

\section{LE BASSIN D'AMORTISSEMENT :}

Le projet initial du bassin rappelait celui de l'usine de Saint-Estève (fig. 16). L'ouvrage compor-

PROJET INITIAL
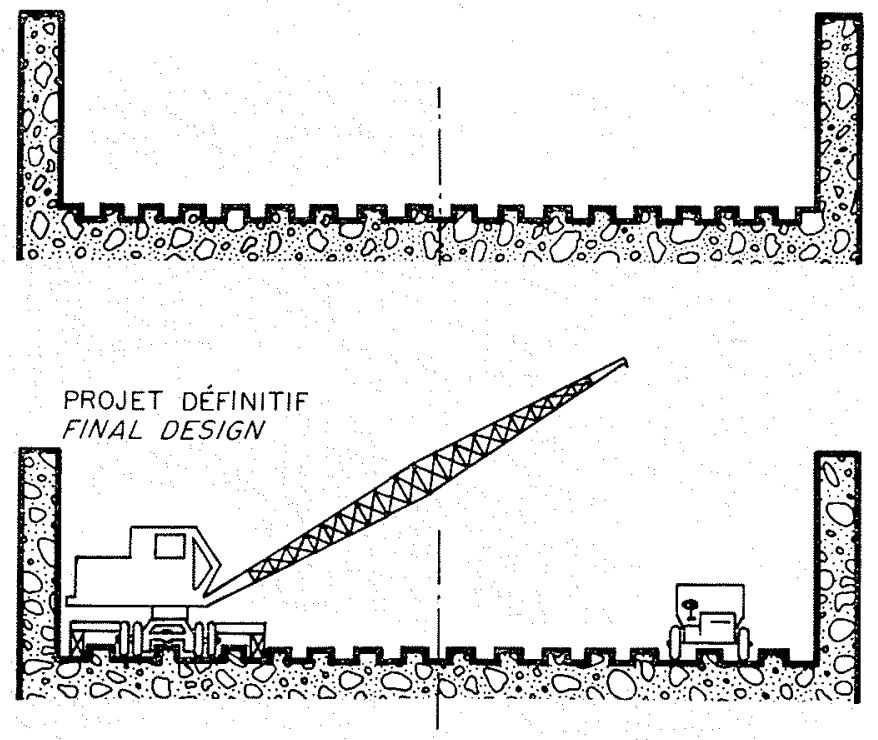

14/ Dispositif de régularisation à guideaux dans le canal de décharge de Salon.

Flow control grooves in the Salon discharge canal.

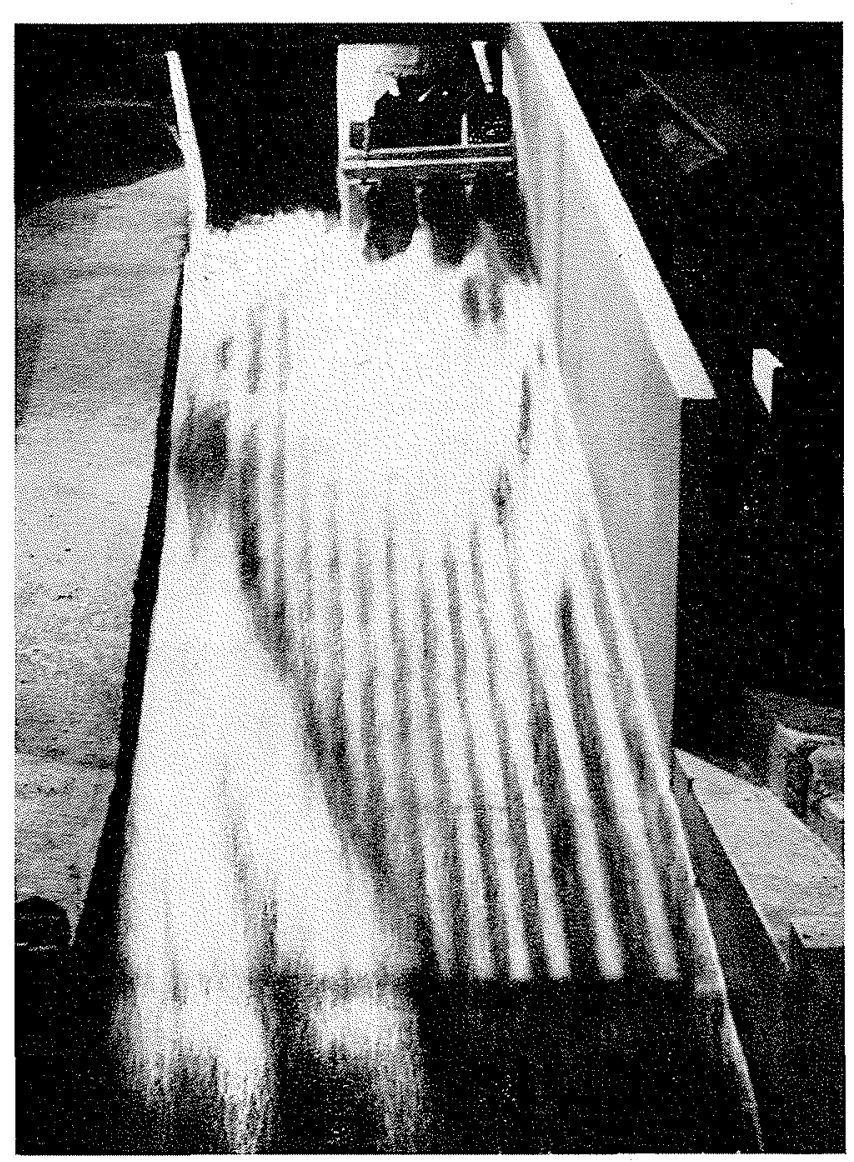

15/ Ecoulement dans le canal de decharge de Salon. Projet définitif.

Flow pattern in the Salon discharge canal. Final design.
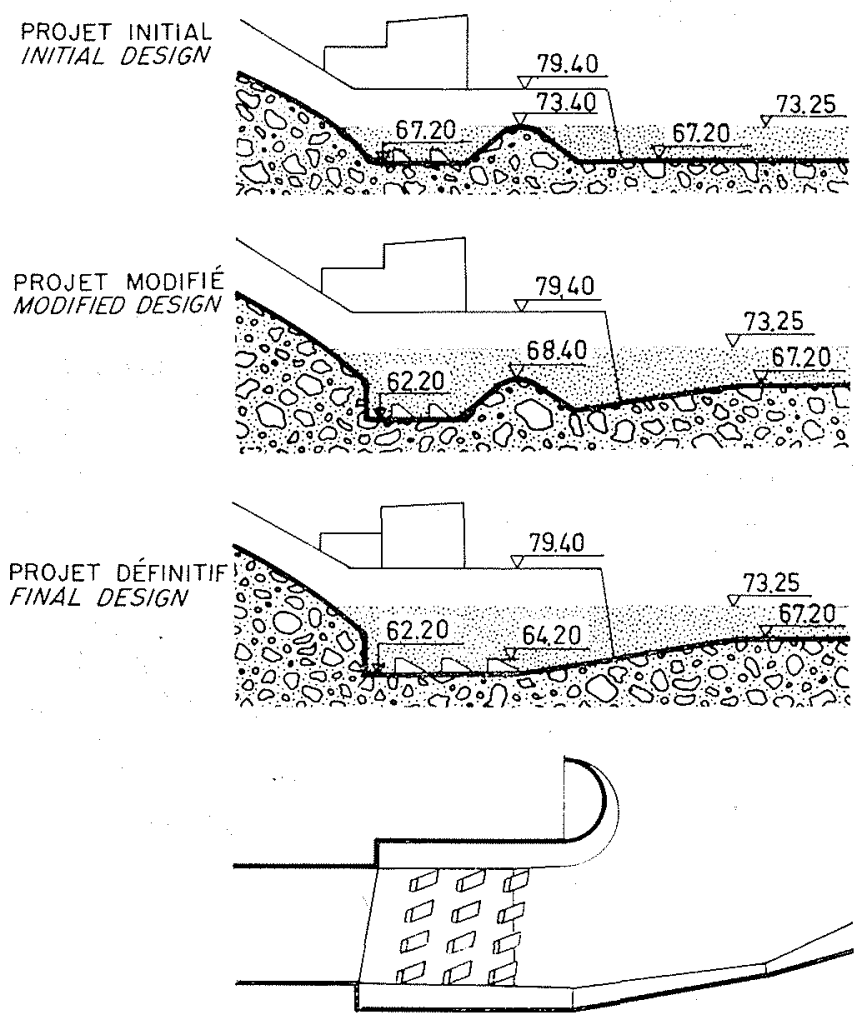

16/ Bassin d'amortissement du déchargeul: de Salon. Salon discharge canal stilling basin. 
tait un radier horizontal à la cote 67,20 (cote du plafond du canal de fuite), limité à l'aval par un seuil calé à la cote 73,40 et muni de deux rangées de 7 dents de $2 \mathrm{~m}$ de hauteur. Les essais ont mis en évidence une dissipation d'énergie insuffisante et une forte agitation de l'eau dans le bassin de restitution, à l'aval du seuil. La première modification du projet a été un abaissement de $5 \mathrm{~m}$ de l'ensemble du bassin et du seuil; les deux rangées de dents ont été conservées; une nelte amélioration des conditions de dissipation d'énergie a été constatée au cours des essais, mais lagitation de la surface du bassin de restitution restait encore inacceptable.

Dans le projet définitif, le plafond du bassin d'amortissement est maintenu à la cote 62,20 ; le
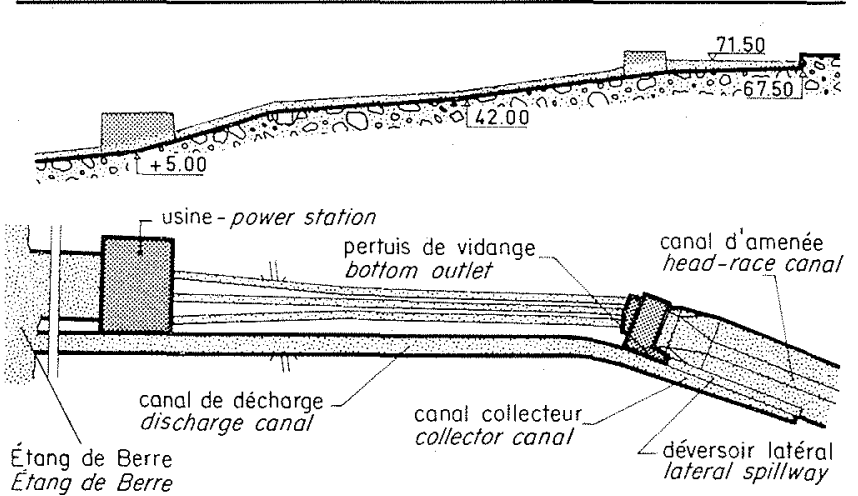

18/ Le dechargeur de Saint-Chamas. Saint-Chamas spillway arrangements.

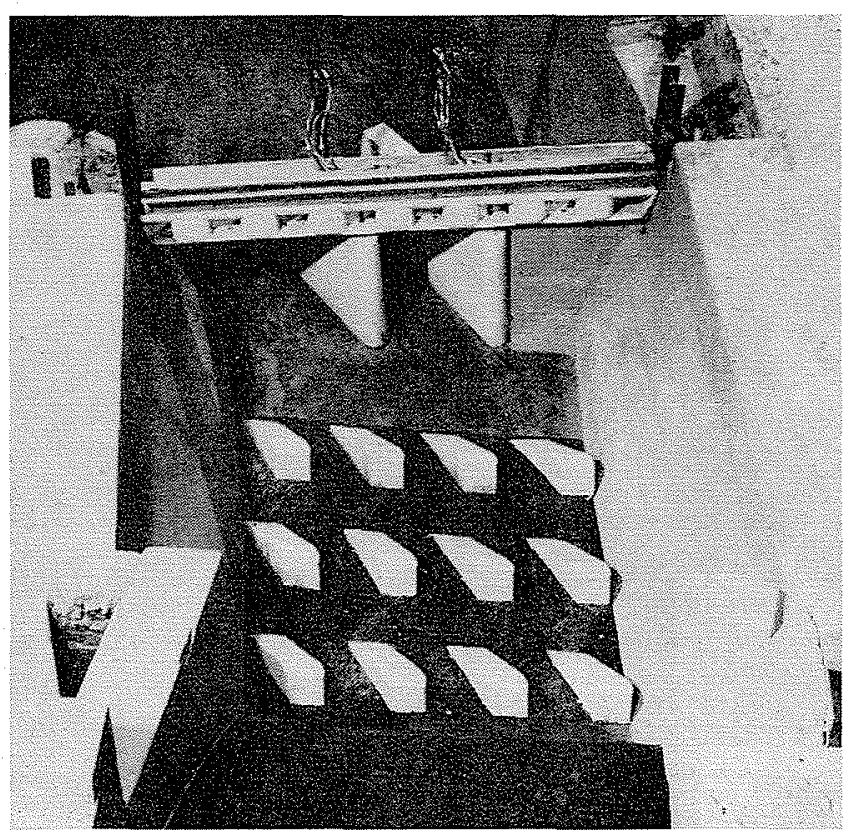

11 Bassin d'amortissement et ouvrage de restitution du déchargeur de Salon. Projet définitif.

Stilling basin and tail-race works for Salon discharge canal. Final design.

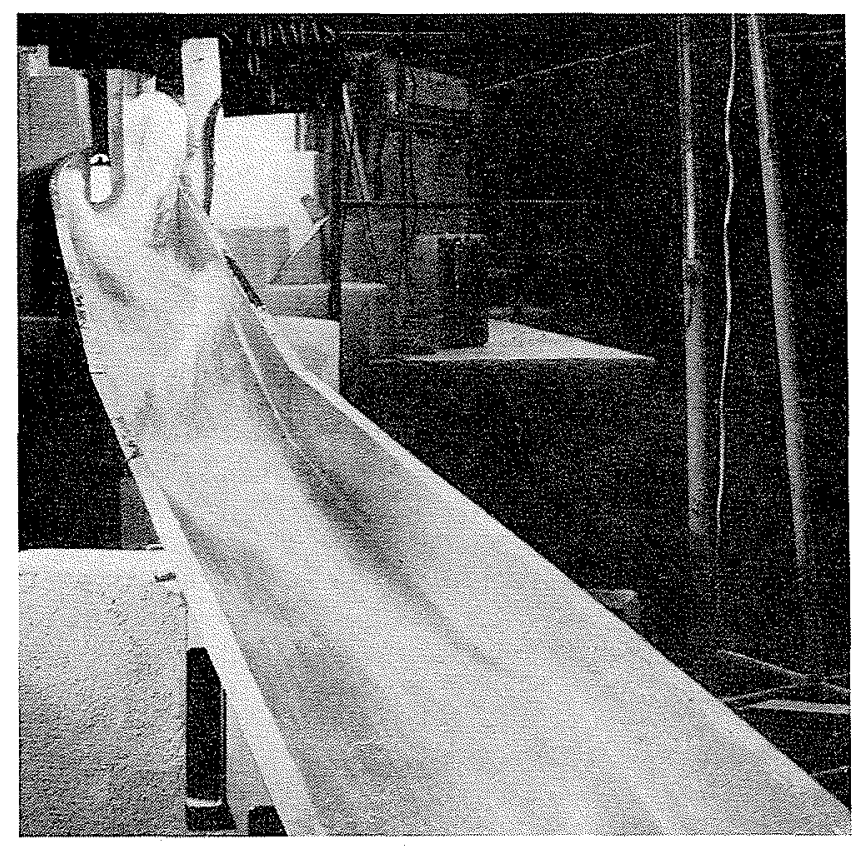

19/ Ecoulement dans le canal de décharge de Saint-Chamas. Projet initial.

Flow pattern in the Saint-Chamas discharge canal. Initial design. seuil de $6,20 \mathrm{~m}$ de hauteur limitant le bassin à l'aval est supprimé et le raccordement avec le plafond du bassin de restitution se fait avec la contre-pente prolongeant celle de la sorlie des aspirateurs de l'usine. Le fond du bassin est équipée de 12 dents inclinées, de $2 \mathrm{~m}$ de hauteur, disposées en trois rangées. Les essais montrent une nette amélioration des conditions d'écoulement dans le bassin de restitution, bien qu'il subsiste une agitation de surface provoquant de légers débordements sur le talus rive droite ef une mauvaise répartition des vitesses dans le canal de fuite.

L'OUVRAgE DE RESTITUTION AU CANAL DE FUTTE :

L'amélioration de la restitution au canal de fuite des eaux de décharge est obtenue par un ouvrage disposé entre le bassin d'anorlissement et le bassin de restitution (fig. 17). Cet ouvrage se compose d'une poutre-masque partiellement en charge, destinée à filtrer l'agitation de surface, et de deux piles guideaux placées sous la poutre et orientées de manière à diriger l'écoulement ver's l'axe du canal de

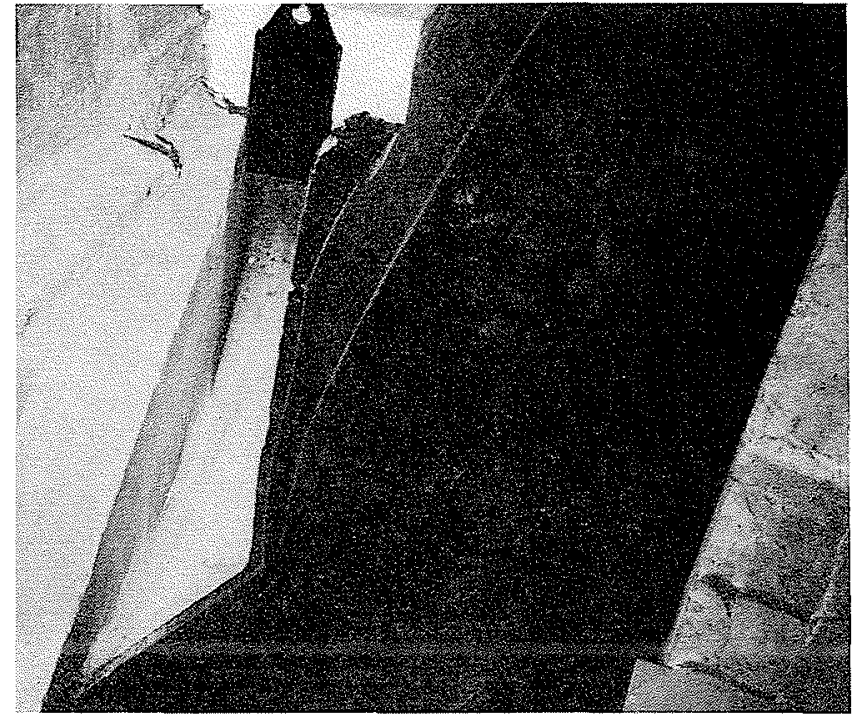

20/ Cuillère du pertuis de vidange de Saint-Chamas. Bottom outlet flip-bucket at Saint-Chamas. 


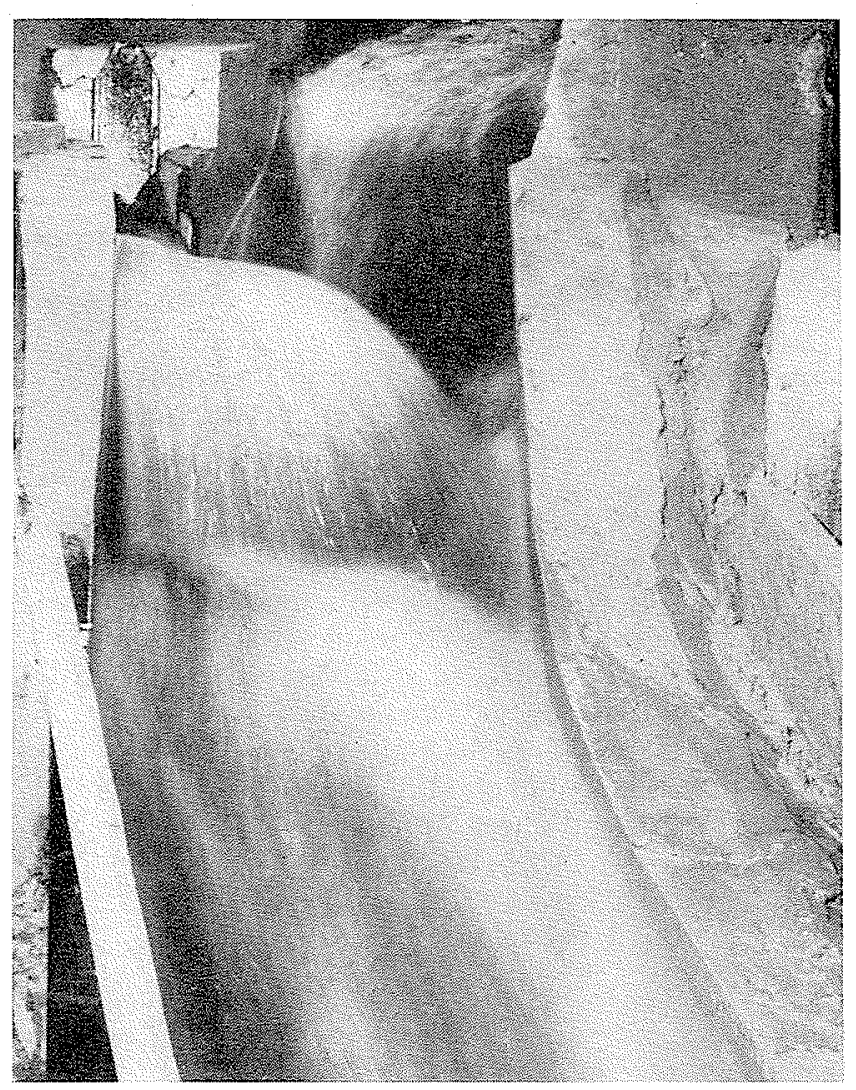

21/ Cuillère du pertuis de vidange de Saint-Chamas en fonctionnement.

Saint-Chamas bottom outlet fip-buciet in operation.

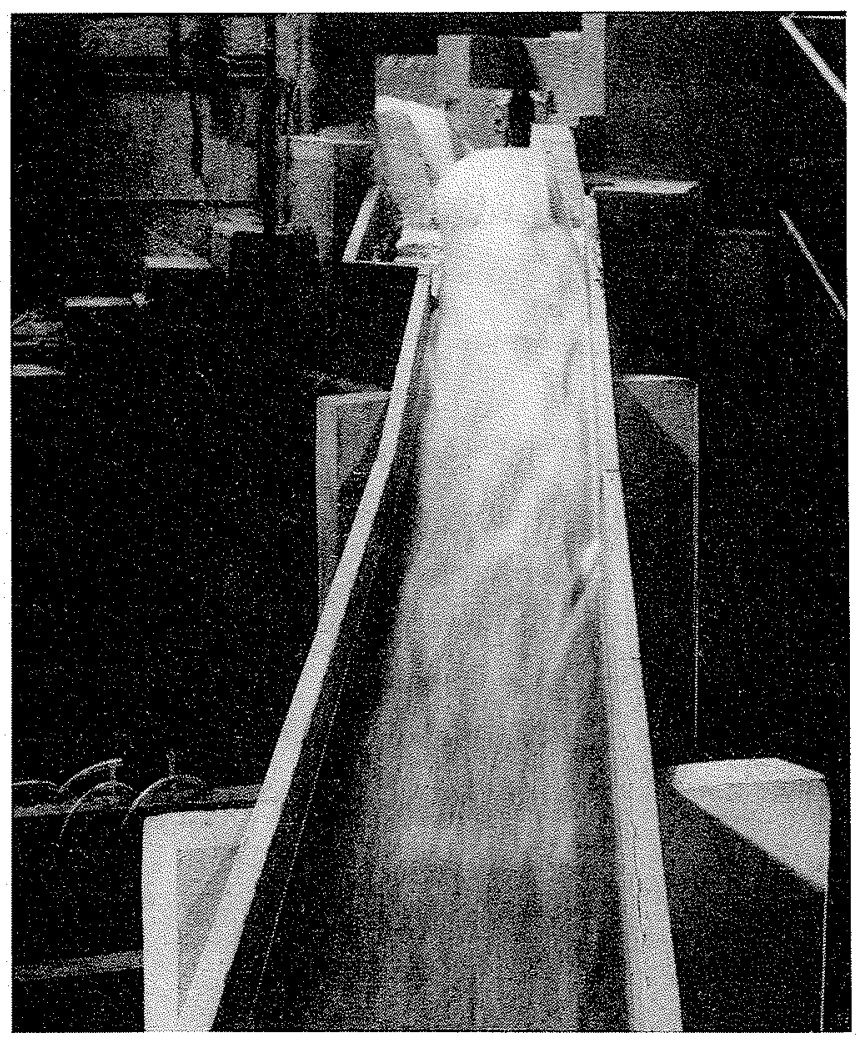

22/ Eeoulement dans le canal de décharge de Saint-Chamas. Projet définitil.

Flow pattern in Saint Chamas discharge canal. Final design. fuite. Les piles délimitent trois passes; la passe rive droite, fermée par un voile de béton, n'est pas utilisée. Huit pertuis de $1 \times 1,5 \mathrm{~m}$ sont réalisés dans la longueur de la poutre pour permettre l'évacuation des corps flottants.

Les ouvrages, poutre, piles, voile de la passe rive droite ont été représentés sur le modèle sans liaison entre eux, de façon à obtenir, par des mesures extensométriques et moyennant certaines hypothèses simplificatrices, la valeur particulière de l'effort exercé par l'écoulement sur chacun d'eux, exception faite du voile. Les résultats des mesures conduisent aux valeurs maximales suivantes:

-- effort horizontal sur la poutre : $50 \mathrm{t}$;

- effort vertical sur chaque appui de la poutre, dirigé de bas en haut :

- $55 \mathrm{t}$ lorsque le déchargeur ne fonctionne pas (poussée hydrostatique),

- $45 \mathrm{t}$ lorsque le déchargeur fonctionne;

- effort longitudinal sur chaque pile: $5 \mathrm{t}$;

- effort transversal sur chaque pile : $25 \mathrm{t}$.

\section{Déchargeur de Saint-Chamas}

Le déchargeur de Saint-Chamas comporte (figure 18);

- un déversoir latéral de sécurité suivi d'un canal collecteur des eaux déversées;

- un pertuis de vidange du canal d'amenée, la restitution directe à l'étang de Berre ne nécessitant pas de pertuis asservis aux groupes de l'usine;

- un canal de décharge à la partie aval duquel se trouve un ouvrage de dispersion, qui a été mis au point au Laboratoire des Milles.

LE DÉVERSOIR ET LE CANAL DE DÉCHARGE :

Le déversoir latéral de sécurité a une longueur de $115 \mathrm{~m}$; il évacue le débit de $250 \mathrm{~m}^{3} / \mathrm{s}$ sous Ia charge de $1,09 \mathrm{~m}$. Le canal de décharge est alimenté de façon normale par le déversoir latéral suivi de son canal collecteur et de façon exceptionnelle par le pertuis de vidange.

Dans le projet initial, le canal collecteur et le pertuis de vidange étaient disposés à côté l'un de l'autre en tête du canal de décharge et ce dernier présentait en plan une courbe de $150 \mathrm{~m}$ de rayon moyen. Les essais ont mis en évidence une très mauvaise alimentation du canal de décharge provoquant la formation d'ondes stationnaires sur toute la longueur de l'ouvrage (fig. 19). Pour le débit maximal, la cote de l'eau au premier point de réflexion de l'onde dépassait de $3 \mathrm{~m}$ la partie supérieure du bajoyer.

A la suite de ces essais, la largeur du canal collecteur a été portée à celle du canal de décharge qui est de $12 \mathrm{~m}$, le pertuis de vidange venant se raccorder latéralement au canal. Les essais de cette nouvelle disposition, effectués d'abord en conservant le rayon de courbure de $150 \mathrm{~m}$ du canal, et ensuite en portant ce rayon à $194 \mathrm{~m}$ ont mis en évidence une légère amélioration de l'écoulement. Des résultats satisfaisants ont toutefois pu ètre obtenus en introduisant un dévers de $10 \%$ dans la courbure en tête du canal et en supprimant le débouché du pertuis de vidange au niveau du canal; on a pour cela surélevé la cote du radier du pertuis de vidange et réalisé une cuillère dont la mise au point a fait l'objet d'une autre série d'essais sur modèle. 


\section{R. LONGUEMARe et L. PUGNet}

LA CUILLERE DU PERTUIS DE VIDANGE :

Les essais sur modèle ont conduit à étudier une cuillère dans le but de répartir uniformément le débit de vidange sur toute la largeur du canal de décharge sans provoquer des rejaillissements trop importants sur les bajoyers. Cette cuillère ne devait en outre, présenter sur le canal de décharge qu'un surplomb de valeur limitée. Plusieurs ouvrages conduisant à des résultats satisfaisants ont été définis, mais la plupart comportaient des surfaces gauches. La cuillère retenue ne se compose que d'éléments plans et d'une surface réglée (fig. 20). La longueur de l'ouvrage projeté est de $13,25 \mathrm{~m}$; le porte-à-faux maximal sur le canal de décharge est de $1,8 \mathrm{~m}$. La partie inférieure de l'ouvrage est profilée de manière à ne pas gêner l'écoulement dans le canal de décharge. Les différents plans successifs constituant le radier de la cuillère, redressent et épanouissent la veine qui alimente le canal de décharge sur toute sa largeur (fig. 21). L'écoulement dans le canal est satisfaisant, qu'il y ait ou non alimentation par le déversoir latéral de sécurité; il n'y a en particulier, en aucun cas, formation d'ondes stationnaires (fig. 22).
Au voisinage de la cuillère et du jet de la vidange, on a donné aux bajoyers du canal de décharge une hauteur suffisante pour éviter les débordements, et un encorbellement de $0,60 \mathrm{~m}$ assure une protection supplémentaire contre les projections.

\section{Conclusion}

L'expérience acquise au cours de la construction et de l'exploitation des déchargeurs des usines de Jouques et de Saint-Estève a contribué à mener à bien les études des aménagements de Salon et de Saint-Chamas. L'expérience de la construction et de l'exploitation de ces derniers pourra sans nul doute être profitable aux études prochaines de l'usine de Mallemort. Toutefois, les considérations précédentes sur les déchargeurs en exploitation et en projel des usines de la. Basse Durance ne sauraient être complètes sans un exposé particulier de l'ouvrage de dissipation d'énergie du déchargeur de SaintChamas qui fait l'objet de la communication suivante. 